\title{
Atypical clinical presentation of COVID-19 infection in residents of a long-term care facility
}

\author{
Hubert Blain ${ }^{1} \cdot$ Yves Rolland $^{2} \cdot$ Athanase Benetos $^{3} \cdot$ Nadia Giacosa $^{1} \cdot$ Mylène Albrand $^{1} \cdot$ Stéphanie Miot $^{1}$. \\ Jean Bousquet ${ }^{4}$
}

Received: 19 May 2020 / Accepted: 29 June 2020 / Published online: 6 October 2020

(c) European Geriatric Medicine Society 2020

\section{Key summary points}

Aim To assess the magnitude of the infection in residents from-and staff working in — a long-term-care facility (LTCF) 7 days after the identification of one resident with confirmed COVID-19 infection and the clinical presentation of the infected residents.

Findings Two weeks after the first case, $50.9 \%$ of residents and $27.5 \%$ of the workers were tested positive for SARS-CoV-2. Diarrhea, fall, delirium, and hypothermia can be initial symptoms of COVID-19 in LTCF residents.

Message The rapid dissemination of a COVID-19 infection in a LTCF may be due to a delay in the diagnosis of the first case, whose presentation could be atypical in residents.

\begin{abstract}
Purpose To assess the magnitude of the infection in residents from—and staff working in —a long-term-care facility (LTCF) 7 days after the identification of one resident with confirmed COVID-19 infection and to assess the clinical presentation of the infected residents.

Methods All residents and staff members of a LTCF were tested for SARS-CoV-2 by real-time reverse-transcriptase polymerase chain reaction on nasopharyngeal swab. Residents were studied clinically 4 weeks after the first COVID diagnosis. Results Thirty-eight of the 79 residents (48.1\%) tested positive for SARS-CoV-2. Respiratory symptoms were preceded by diarrhea (26.3\%), a fall (18.4\%), fluctuating temperature with hypothermia $(34.2 \%)$ and delirium in one resident. Respiratory symptoms, including cough and oxygen desaturation, appeared after those initial symptoms or as the first sign in $36.8 \%$ and $52.2 \%$, respectively. At any time of the disease, fever was observed in $65.8 \%$. Twelve deaths occurred among the COVID19 residents. Among the 41 residents negative for SARS-CoV-2, symptoms included cough (21.9\%), diarrhea (7.3\%), fever (21.9\%), hypothermia (9.7\%), and transient hypoxemia (9.8\%). No deaths were observed in this group. $27.5 \%$ of the workers were also COVID-19 positive.

Conclusion The rapid dissemination of the COVID-19 infection may be explained by the delay in the diagnosis of the first cases due to atypical presentation. Early recognition of symptoms compatible with COVID-19 may help to diagnose COVID-19 residents earlier and test for SARS-CoV-2 symptomatic and asymptomatic staff and residents earlier to implement appropriate infection control practices.
\end{abstract}

Keywords COVID-19 $\cdot$ Testing for SARS-CoV-2 $\cdot$ Long-term-care facility $\cdot$ rRT-PCR

Hubert Blain

h-blain@chu-montpellier.fr

1 Department of Geriatrics, Centre Antonin Balmes, Pôle de Gérontologie du Centre Hospitalier Universitaire de Montpellier, Montpellier University Hospital, Montpellier University, 39 Avenue Charles Flahault, 34295 Montpellier Cedex 5, France

2 Gérontopôle de Toulouse, INSERM 1027, 31059 Toulouse, France
3 Department of Geriatrics, CHRU de Nancy and Inserm DCAC, Université de Lorraine, Nancy, France

4 Department of Dermatology and Allergy, Berlin Institute of Health, Comprehensive Allergy Center, Charité, Universitätsmedizin Berlin, Humboldt-Universität zu Berlin, Berlin, Germany 


\section{Introduction}

Older adults and those with underlying chronic medical disorders tend to be more susceptible to severe SARSCoV-2 (COVID-19) [1, 2]. The population residing in longterm-care facilities (LTCFs) generally includes those who are both old and suffering from multiple comorbidities. A recent investigation conducted after the identification of a confirmed case of COVID-19 in a skilled nursing facility demonstrated the magnitude of the problem [3]. In this study, most of the residents with COVID-19 had respiratory illness at the moment of the diagnosis and the fatality rate was $33.7 \%$. In another study conducted in younger residents of a skilled nursing facility, more than half of COVID-19 residents were judged asymptomatic before testing [4]. It is possible that older residents in LTCFs have specific initial atypical COVID-19 symptoms that need to be recognized rapidly.

Three residents living in a LTCF were admitted for severe non-respiratory symptoms. Case 1: March 3, 2020: diarrhea with dehydration and a severe health status impairment. Case 2: March 5, 2020: fall with a wrist fracture in a woman without significant balance disturbance. Case 3: March 6, 2020: delirium, hypothermia, and hypotension in a woman with previous moderate cognitive impairment. The three residents all developed respiratory symptoms (cough with fever and dyspnea) 7-10 days after their admission to hospital and nasopharyngeal swab test confirmed COVID-19 diagnosis. All three patients died 5-7 days after diagnosis. As soon as the diagnosis was made in the first resident, infection prevention and control (ICP) measures were immediately adopted in the LTCF.

The study attempted to assess the prevalence of COVID19 infection in residents and staff members of the LTCF 7 days after the COVID-19 diagnosis of the first resident. The longitudinal symptoms of the residents were also assessed for 4 weeks.

\section{Methods}

Following local guidance, SARS-CoV-2 real-time reversetranscriptase polymerase chain reaction (rRT-PCR) was used on nasopharyngeal swab tests in all 79 residents and 40 health workers and on the stools of five of the residents with diarrhea. This was an observational study approved by the Montpellier University Hospital Institutional Review Board (IRB-MTP_2020_06_202000534). Symptoms in the 14 days before positive testing and until March 30 were recorded in all residents.

\section{Results}

Among the 79 residents, $38(48.1 \%)$ were positive for SARS-CoV-2 on March 18-20, 2020. Respiratory symptoms were preceded by diarrhea in $10(26.3 \%)$ cases, a fall in 7 (18.4\%), and hypothermia in 13 (34.2\%). Respiratory symptoms appeared after those symptoms or as the first sign in 24 residents $(63.2 \%)$, including a dry cough in 14 (36.8\%) and hypoxemia in $21(55.2 \%)$. At any time of the disease, fever was observed in 25 residents $(65.3 \%)$, most often alternating with hypothermia in 13 residents (34.2\%). Three residents had no symptoms at the time of testing (7.9\%), but they developed fever and cough 3-10 days later. Besides the first three deaths in the hospitalized residents, nine other COVID-19-positive residents died during the follow-up period, always less than 10 days after initial testing. This represents a total fatality rate of $31.6 \%(12 / 38)$ in the residents tested positive for SARS-CoV-2. The residents who died had severe hypoxemia in 12 cases (100\%), hectic fever in $10(83.3 \%)$, and diarrhea in $9(75.0 \%)$. In all 12 cases, maximum oxygen supply was not sufficient to restore normal blood oxygen saturation. All of these residents benefited from optimal end-of-life care, three in hospital and six in the LTCF.

Among the 41 residents tested negative for SARS-CoV-2, $22(53.6 \%)$ had new symptoms in the 14 days before testing, including cough in 9 cases (21.9\%), diarrhea in 3 (7.3\%), fever in 2 (4.8\%), hypothermia in 2 (4.8\%), and dyspnea with transient hypoxemia in 4 (9.8\%). No deaths were observed in this group (Table 1).

In the five residents with diarrhea (two tested positive and three tested negative for SARS-CoV-2), all rectal swab tests by rRT-PCR were negative for SARS-CoV-2.

On March 19, 2020, 11 of the 40 staff members (27.5\%) were tested positive for SARS-CoV-2. All of them were asymptomatic or had minor complaints, such as asthenia, in the 14 days before testing. On March 30, 2020, the progression of health status of all staff members tested positive for SARS-CoV-2 was favorable.

\section{Discussion}

The present case investigation provides novel insight into COVID-19 infection in old-age adults in LTCFs and extends the papers of McMichael et al. [3] and Arons et al. [4].

There were around 50\% of COVID-19 infected residents and $27.5 \%$ of health care workers. This confirms that a few days after a first case of confirmed COVID-19 in a LTCF, the number of other residents and staff members linked to the first case can be very high [3]. 
Table 1 Demographic and clinical characteristics

\begin{tabular}{|c|c|c|c|}
\hline & $\begin{array}{l}\text { COVID+ resi- } \\
\text { dents, } N=38 \\
n(\%)\end{array}$ & $\begin{array}{l}\text { COVID- resi- } \\
\text { dents, } N=41 \\
N(\%)\end{array}$ & $P$ value** \\
\hline Age (years)* & $89.2(5.6)$ & $87.3(9.8)$ & 0.44 \\
\hline Gender, male & $8(29.6)$ & $13(50.0)$ & 0.13 \\
\hline \multicolumn{4}{|c|}{ Symptoms before respiratory symptoms } \\
\hline $\begin{array}{l}\text { Fluctuating tempera- } \\
\text { ture with hypother- } \\
\text { mia }\end{array}$ & $13(34.2)$ & $2(4.8)$ & $<0.01$ \\
\hline Diarrhea & $10(26.3)$ & $3(7.3)$ & 0.06 \\
\hline Fall & $7(18.4)$ & $0(0)$ & $<0.01$ \\
\hline \multicolumn{4}{|l|}{ Respiratory symptoms } \\
\hline Cough & $14(36.8)$ & $9(21.9)$ & 0.29 \\
\hline Oxygen desaturation & $21(55.2)$ & $4(9.8)$ & $<0.01$ \\
\hline \multicolumn{4}{|c|}{ Symptoms at any time of the 27 -day follow-up } \\
\hline Fever & $25(65.8)$ & $9(21.9)$ & $<0.01$ \\
\hline Hypothermia & $13(34.2)$ & $4(0.7)$ & $\mathbf{0 . 0 3}$ \\
\hline Diarrhea & $15(39.5)$ & $10(24.4)$ & 0.29 \\
\hline Fall & $9(23.7)$ & $2(4.9)$ & 0.07 \\
\hline Severe myalgia & $10(26.3)$ & $1(2.4)$ & $<0.01$ \\
\hline Deaths & 13 (31.6) & $0(0)$ & $<0.01$ \\
\hline
\end{tabular}

*Continuous variable age is expressed as mean (SD); **P for Chisquare test (or Fisher's exact test if Chi-square was not a valid test) for categorical variables and Student test for continuous variables

The rapid dissemination of COVID-19 may, at least in part, be explained by the delay in the diagnosis in the first residents and, therefore, in the implementation of appropriate infection control practices (ICPs). The first possible reason for this delay may be related to asymptomatic forms of COVID-19 at the time of testing, such as in younger people [5], those patients representing $7.3 \%$ of residents tested positive for SARS-CoV-2 of our sample vs $56 \%$ in another study conducted by Arons in a skilled nursing facility [4]. All of these cases developed typical COVID-19 symptoms after testing in the present study which is in line with previous studies conducted in younger patients [4-6]. The second possible reason of the diagnosis delay may be related to atypical symptoms preceding the respiratory symptoms and fever, including diarrhea, hypothermia, or delirium. Another interesting feature is the relatively common fall rate as the first symptom. Since COVID-19 induces severe fatigue, it can be expected that falls can be common in residents with little or no risk of fall. This symptom needs to be considered as a possible prodromal symptom of COVID-19 during the epidemic in LTCF residents. Diarrhea prevalence has been shown to increase in the later stage of the COVID-19 disease and is often associated with COVID-19 infectious stools [7]. The present study suggests that diarrhea can be a prodromal sign of COVID-19, with stools that were not infectious in five of our patients with diarrhea.
The second factor that may explain, at least partly, the rapid and large COVID-19 outbreak in this LTCF is the high prevalence of staff members positive for SARS-CoV-2. The present data strongly support those of McMichel et al. [3], underlying the involuntary role of the health workers and visitors in the COVID-19 spread within this facility. They led us to take the decision to prohibit visits in the LCTF and to systematically screen for SARS-CoV-2 all staff members and residents (even asymptomatic) as soon as a case of COVID-19 was confirmed in the first resident.

In addition to appropriate ICP measures, the strategies of systematic testing residents with typical and atypical COVID-19 symptoms to diagnose early the first case, and then, of early testing for SARS-CoV-2 all residents, visitors, and staff members, even asymptomatic, may be useful in controlling COVID-19 outbreaks in LTCFs, and may contribute to decreasing the case fatality ratio of the disease.

In conclusion, the present study shows that the rapid dissemination of a COVID-19 infection in a LTCF may be due to a delay in the diagnosis of the first case, whose presentation could be atypical. An early recognition of atypical symptoms compatible with COVID-19 may help diagnose the first case earlier, allow early diagnosis of COVID-19 in staff, visitors, and other residents, and implement earlier targeted ICPs in the LTCF.

Acknowledgements The authors thank Audrey Jaussent for help in data analysis, Anna Bedbrook and Fabienne Portejoie for editorial assistance and all residents and health care workers of the long-term care facility.

Funding This research did not receive any funding from agencies in the public, commercial, or not-for-profit sectors.

\section{Compliance with ethical standards}

Conflict of interest The authors declare that they have no conflict of interests.

Ethical approval This was an observational study approved by the Montpellier University Hospital Institutional Review Board (IRBMTP_2020_06_202000534).

Informed consent For this type of study, formal consent is not required.

\section{References}

1. Guan WJ, Ni ZY, Hu Y, Liang WH, Ou CQ, He JX et al (2020) Clinical characteristics of coronavirus disease 2019 in China. N Engl J Med. https://doi.org/10.1056/NEJMc2005203

2. D'Adamo H, Yoshikawa T, Ouslander JG (2020) Coronavirus disease 2019 in geriatrics and long-term care: the ABCDs of COVID-19. J Am Geriatr Soc. https://doi.org/10.1111/jgs.16445

3. McMichael TM, Currie DW, Clark S, Pogosjans S, Kay M, Schwartz NG et al (2020) Epidemiology of Covid-19 in a 
long-term care facility in King County, Washington. N Engl J Med. https://doi.org/10.1056/NEJMoa2005412

4. Arons MM, Hatfield KM, Reddy SC, Kimball A, James A, Jacobs JR et al (2020) Presymptomatic SARS-CoV-2 infections and transmission in a skilled nursing facility. N Engl J Med. https:// doi.org/10.1056/NEJMoa2008457

5. Mizumoto K, Kagaya K, Zarebski A, Chowell G (2020) Estimating the asymptomatic proportion of coronavirus disease 2019 (COVID-19) cases on board the Diamond Princess cruise ship, Yokohama, Japan, 2020. Euro Surveill. https://doi. org/10.2807/1560-7917.ES.2020.25.10.2000180

6. Luo SH, Liu W, Liu ZJ, Zheng XY, Hong CX, Liu ZR et al (2020) A confirmed asymptomatic carrier of 2019 novel coronavirus
(SARS-CoV-2). Chin Med J. https://doi.org/10.1097/CM9.00000 00000000798

7. Jin X, Lian JS, Hu JH, Gao J, Zheng L, Zhang YM et al (2020) Epidemiological, clinical and virological characteristics of 74 cases of coronavirus-infected disease 2019 (COVID-19) with gastrointestinal symptoms. Gut. https://doi.org/10.1136/gutjn $1-2020-320926$

Publisher's Note Springer Nature remains neutral with regard to jurisdictional claims in published maps and institutional affiliations. 